\title{
Estrategia tecnológica para nivelar los presaberes matemáticos en la educación superior
}

\section{Karol Lisette Rueda-Gómez ${ }^{\mathrm{a}}$ y Luis J. Rodríguez-Muñiz ${ }^{\mathrm{b}}$}

aUniversidad de Oviedo (España) y Unidades Tecnológicas de Santander (Colombia) krueda@correo.uts.edu.co, ๖Universidad de Oviedo (España) luisj@uniovi.es

\begin{abstract}
Information and Communication Technologies (ICTS) in the educational context allow using digital spaces using innovative and creative methodologies in order to reinforce students' learning in different knowledge areas. Additionally, high dropout and repetition rates in mathematical college courses in the first semesters highlight, among other possible reasons, the freshmen's gap in the mathematics prerequisites. The goal of the present study is to implement a pilot experience by using KHAN ACADEMY with freshmen in a Higher Education Institution in Colombia, with the aim of establishing strategies to improve academic performance of freshmen in mathematics. Conclusions show that it is possible to offer to the freshmen a virtual training to reinforce their mathematical prerequisites by using KHAN ACADEMY, and by assuming the active support of the teaching staff.
\end{abstract}

Keywords: College freshmen, higher education, KHAN ACADEMY, mathematics prerequisites.

\section{Resumen}

Las Tecnologías de la Información y la Comunicación (TIC) permiten utilizar en el ámbito educativo espacios digitales que poseen metodologías innovadoras y creativas para potenciar el aprendizaje de los estudiantes en diferentes áreas del conocimiento. De otro lado, las altas tasas de deserción y repetición en las asignaturas del área de matemáticas en los primeros semestres académicos de la educación superior evidencian, entre otras posibles causas, el bache formativo en los presaberes matemáticos de los estudiantes de nuevo ingreso. El objetivo de este estudio es implementar una prueba piloto del uso de la plataforma online KHAN ACADEMY en estudiantes de nuevo ingreso de una Institución de Educación Superior (IES) colombiana con el fin de fomentar estrategias de mejora que favorezcan los objetivos académicos de los estudiantes en el área de matemáticas. Se concluye que es posible ofrecer a los estudiantes de nuevo ingreso un entrenamiento virtual en el que pueden reforzar los presaberes matemáticos del bachillerato mediante el uso de KHAN ACADEMY, contando con el apoyo activo de los docentes.

Palabras clave: Educación superior, estudiantes de nuevo ingreso, KHAN ACADEMY, presaberes matemáticos.

\section{Introducción}

Las TIC son importantes dentro de las políticas públicas de cada país puesto que promueven el desarrollo de la sociedad a través del mejoramiento de la calidad de vida, reduciendo las brechas de desigualdad (Martínez, Pérez \& Martínez, 2016). En el ámbito educativo se destacan también las Tecnologías del Aprendizaje y el Conocimiento (TAC), enfocadas a propiciar un entorno formativo personalizado mediante los usos didácticos que las TIC tienen para enriquecer el proceso de enseñanza y aprendizaje. Las TAC se han convertido en herramientas facilitadoras y dinamizadoras del saber, puesto que permiten 
la descentralización del conocimiento y la creación de metodologías personalizadas de enseñanza en espacios innovadores de aprendizaje (Utomo, Bon \& Hendayun, 2018). No obstante, la incorporación de las TAC dentro del proceso formativo reglado en educación superior en Colombia es escasa, señalándose como algunas de las causas la falta de capacitación por parte de los docentes o los altos costos que genera su utilización (Valdés, Angulo, Urías, García \& Mortis, 2011).

También en el contexto colombiano, los resultados de las diversas pruebas que se han realizado nacional e internacionalmente muestran que los estudiantes colombianos tienen grandes dificultades en el desarrollo de habilidades, particularmente en destrezas matemáticas evidenciando un retraso escolar de casi dos años (Murcia \& Henao, 2015). Así, los resultados de la prueba PISA de la Organización para la Cooperación y el Desarrollo Económicos (OCDE, 2019) señalan que la media obtenida en competencia matemática por los estudiantes colombianos fue de 391, valor que se encuentra 98 puntos por debajo de la media de la OCDE. Además, el informe evidencia que alrededor del $40 \%$ de los estudiantes colombianos muestran un bajo nivel de desempeño en el área de matemáticas.

Del mismo modo, la prueba de competencias Saber 11, implementada para medir la calidad educativa de los colombianos al culminar los estudios de bachillerato, evidencia que los estudiantes de nuevo ingreso llegan a la educación superior con niveles de desempeño bajo en el área de matemática, lo que provoca altos índices de deserción y repetición en los primeros semestres académicos (Rodríguez, Gómez \& Ariza, 2014). Este fenómeno del bajo rendimiento en matemáticas de estudiantes de nuevo ingreso no es ajeno a España, y las universidades han desarrollado distintas estrategias para intentar reducirlo (Díaz \& Rodríguez-Muñiz, 2013; Rodríguez-Muñiz \& Díaz, 2015). Igualmente, las estadísticas del Sistema para la Prevención de la Deserción de la Educación Superior, SPADIES, en Colombia (MEN, 2016) indican que el mayor índice de deserción en las IES colombianas se presenta debido a la falta de presaberes y fundamentación básica desde la educación media en competencias generales (40.5\%) y al bajo rendimiento académico obtenido en las asignaturas del área de matemáticas y ciencias naturales en los primeros semestres académicos $(47.0 \%)$.

\subsection{Planteamiento del problema}

Las Unidades Tecnológicas de Santander (UTS), IES colombiana, deben abordar la anterior problemática, pero con un valor agregado y es que, a diferencia de las demás IES colombianas, las UTS, en coherencia a su misión social, en el proceso de selección de estudiantes no exigen examen de admisión ni puntaje mínimo en las pruebas de Estado. Lo anterior, favorece la inclusión social y facilita el acceso del estudiantado, pero genera un sobresfuerzo institucional en fortalecer las destrezas básicas de los estudiantes en el área de matemática. Por tanto, institucionalmente se está planteando si la posibilidad de un aprendizaje más autónomo a partir de la incorporación de las TIC y las TAC podría servir como factor reductor de este problema. Dada la vocación de la institución, se apuesta por tecnologías de libre acceso, por ejemplo: GEOGEBRA, SYMBOLAB, Moocs, MOODLE, KAHOOT, KHAN ACADEMY, entre otras. En particular, en esta investigación nos vamos a centrar en KHAN ACADEMY, que es una herramienta tecnológica online donde se dispone de recursos educativos en áreas del conocimiento como matemática, biología, química, física, computación, humanidades, economía, finanzas e historia. Los cursos de KHAN ACADEMY van dirigidos a todos los niveles educativos y se ofertan de forma gratuita (Ramírez \& Vizcarra, 2016).

El proceso formativo en KHAN ACADEMY se basa en un sistema de aprendizaje automático que genera una ruta formativa personalizada. Dependiendo de los resultados obtenidos en el examen diagnóstico el sistema determina debilidades y fortalezas. Mientras el estudiante trabaja en la plataforma es evaluado periódicamente a través de los llamados "Desafí s de Dominio" para asegurar que se construya una base sólida. Cabe agregar que el sistema de misiones genera para cada habilidad, ejercicios evaluados

(cc)) EY-NC-ND 2020, Universitat Politècnica de València 
considerando el nivel de desarrollo, clasificándolos en función de la necesidad de mayor o menor práctica, e introduciéndolos posteriormente de manera aleatoria y continua en el "Desafí de dominio" (Dicheva, Dichev, Agre \& Angelova, 2015).

\section{Objetivos}

A partir de lo expuesto en la anterior sección, se evidencia la necesidad que tienen las UTS de fomentar mecanismos que fortalezcan y aseguren los presaberes matemáticos en los estudiantes de nuevo ingreso con el fin de disminuir el bache formativo y reducir la tasa de reprobación en las asignaturas del área de matemáticas (Aguilar, 2014). Cabe resaltar la oportunidad que las TAC nos ofrecen para dar una posible solución a la problemática del bache formativo en los presaberes del área de matemáticas que presentan los estudiantes de nuevo ingreso mediante el uso de la plataforma de aprendizaje artificial KHAN ACADEMY. En consecuencia, la pregunta que se plantea es: ¿pueden los estudiantes que ingresan a primer semestre académico de la educación superior fortalecer sus destrezas matemáticas mediante la utilización de la plataforma KHAN ACADEMY?

La respuesta a la pregunta la buscaremos a través del siguiente objetivo general: implementar un entrenamiento virtual en estudiantes de nuevo ingreso con el fin de fortalecer los presaberes matemáticos. A su vez, este objetivo lo desglosaremos en cuatro objetivos específicos:

- Objetivo 1: Diseñar el curso mediante la utilización de diferentes TIC de acceso libre

- Objetivo 2: Implementar el entrenamiento virtual en tres periodos academicos.

- Objetivo 3: Analizar y comparar los resultados obtenidos en las implementaciones del curso.

- Objetiivo Objetivo 4: Medir el desempeño en el curso, considerando la variable docente.

\section{Desarrollo de la innovación}

La plataforma KHAN ACADEMY ofrece diversos cursos, cada curso esta constituido por un eje temático principal y subtemas. En cada subtema el estudiante dispone de guías interactivas, videos y ejercicios con verificación instantánea de la respuesta y la opción de solicitar pistas indicando el paso a paso de la solución del ejercicio. En la primera etapa de la investigación se eligió el curso "Fundamentos de álgebra", compuesto por 104 habilidades distribuidas así: fundamentos (31 habilidades), expresiones algebraicas (10 habilidades), expresiones con exponentes (11 habilidades), cuadráticas y polinomios (12 habilidades),ecuaciones lineales e inecuaciones (14 habilidades), sistemas de ecuaciones 2x2 (7 habilidades), geometría y ecuaciones (6 habilidades), gráficas de línea y pendiente (13 habilidades). Además, se creó la página web con las instrucciones del proceso de matrícula y desarrollo del curso, que está disponible en $<$ https://preinuts.es.tl $>$.

En la segunda etapa, se extendió la invitación a los estudiantes de nuevo ingreso a realizar el curso mediante correos electrónicos y llamadas telefónicas. También, se creó un grupo de WHATSAPP con los docentes de "Matemática básica" y "Álgebra I" para apoyar la implementación del curso desde el aula de clase.

En la tercera etapa se generaron los reportes desde la plataforma KHAN ACADEMY y se analizaron los datos con el software SPSS.

\section{Resultados}

La primera implementación del curso virtual denominado PREIN contó con la participación de 799 estudiantes pertenecientes a los diferentes programas académicos y sedes de las UTS. La Figura 1 muestra el porcentaje de avance en el entrenamiento en línea obtenido por los estudiantes, en el segundo 
periodo academico de 2018, evidenciando que el $28 \%$ de los estudiantes lograron superar el $50 \%$ de avance total en el curso.

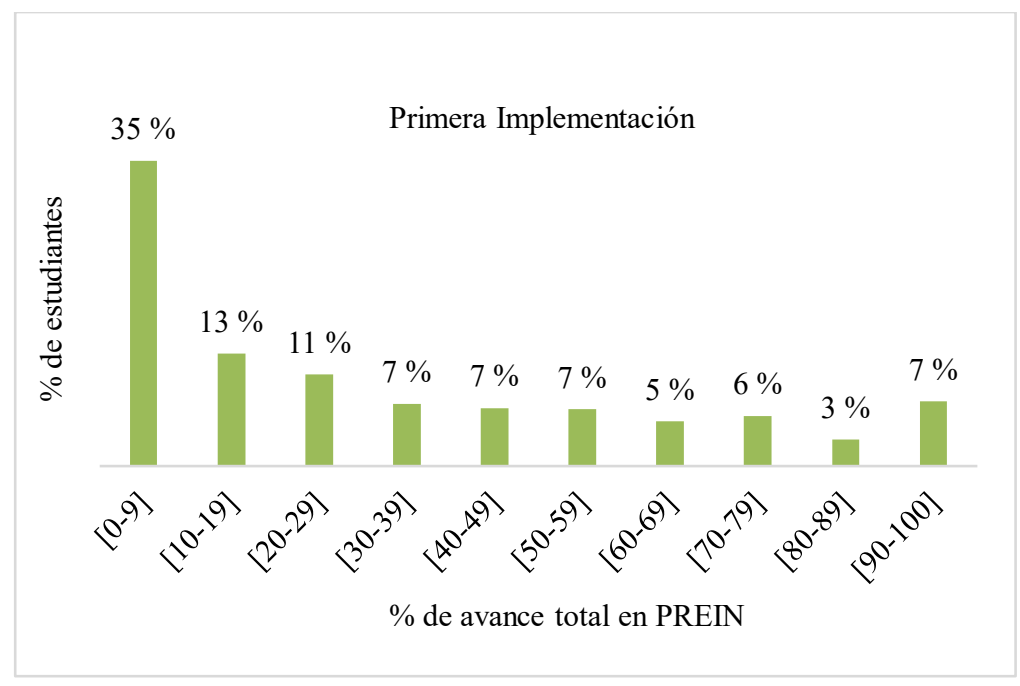

Fig. 1 Porcentaje de avance en el curso PREIN primera implementación

La segunda implementación contó con 1947 estudiantes procedentes de diferentes programas académicos y sedes de las UTS. La Figura 2 muestra el porcentaje de avance en el entrenamiento en línea obtenido por los estudiantes, en el primer periodo académico de 2019. Como se comprueba, el $41 \%$ de los estudiantes lograron superar el $50 \%$ de avance total en el curso.

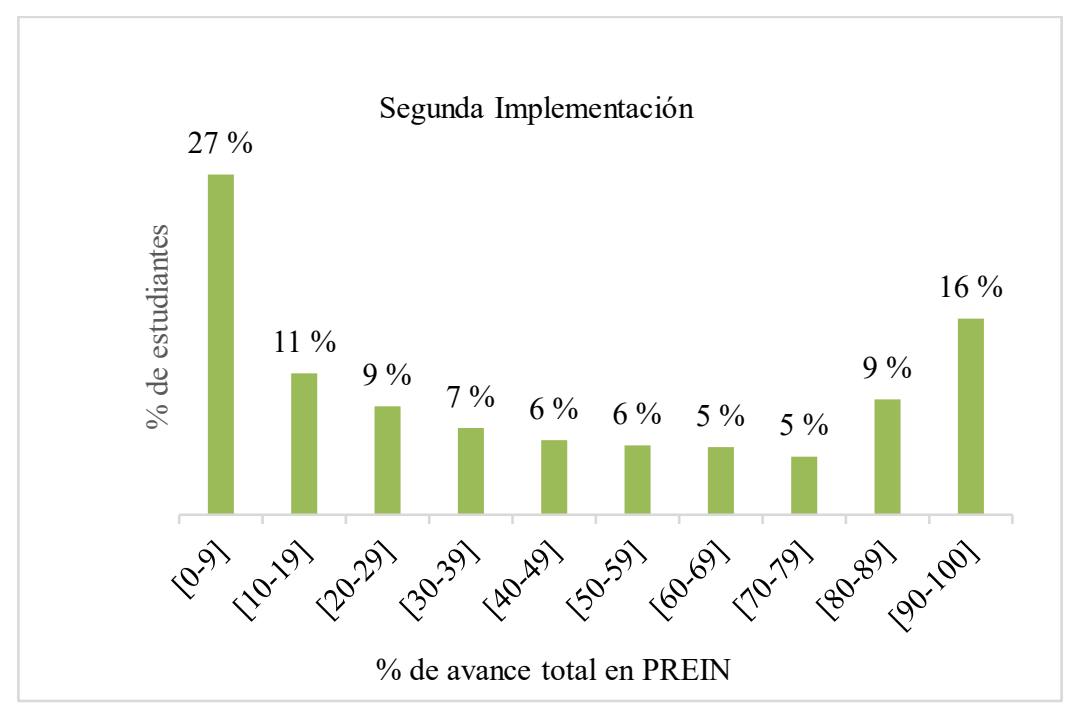

Fig. 2 Porcentaje de avance en el curso PREIN segunda implementación

La tercera implementación contó con la participación de 1986 estudiantes pertenecientes a los diferentes programas académicos y sedes de las UTS. La Figura 3 muestra el porcentaje de avance en el entrenamiento en línea obtenido por los estudiantes, en el segundo periodo académico de 2019, como se aprecia, el $62 \%$ de los estudiantes lograron superar el $50 \%$ de avance total en el curso. 


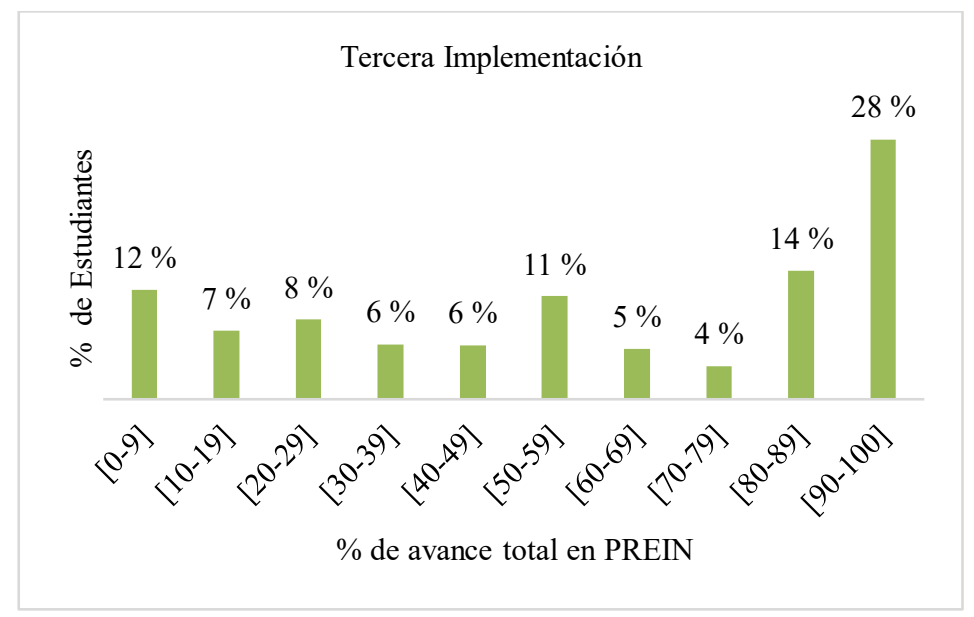

Fig. 3 Porcentaje de avance en el curso PREIN tercera implementación

En cuanto al número de estudiantes matriculados y el porcentaje de avance en el desarrollo del curso los resultados muestran un incremento favorable semestre a semestre. De igual manera, en la Tabla 1 se aprecia de forma detallada el incremento en el porcentaje de avance promedio obtenido por los estudiantes en el curso y en cada una de las unidades temáticas que lo conforman. Teniendo en cuenta que la realización del curso no implicaba un orden, es decir, el estudiante podía iniciar por el desarrollo de la unidad 8 sin haber desarrollado las otras unidades, se evidencia mayor porcentaje de avance en las unidades 1 y 2 y menor porcentaje de avance en las unidades 4,5 y 6 , es decir, los estudiantes muestran mayor dominio en los ejes temáticos de las unidades 1 "Fundamentos" y 2 "Expresiones algebraicas" y menor dominio en los ejes temáticos de las unidades 4 "Gráfica de línea y pendiente" , 5 "Sistemas de ecuaciones" y 6 "Expresiones con exponentes".

Tabla 1. Desempeño en el curso PREIN durante las tres implentaciones.

\begin{tabular}{cccc}
\hline & 1a Implementación & $2^{\mathrm{a}}$ Implementación & $3^{\mathrm{a}}$ Implementación \\
& $2018-\mathrm{II}$ & $2019-\mathrm{I}$ & $2019-\mathrm{II}$ \\
& $\mathrm{N}=799$ & $\mathrm{~N}=1947$ & $\mathrm{~N}=1986$ \\
\hline Media del curso & $31.52 \%$ & $42.32 \%$ & $58.39 \%$ \\
Unidad 1: Fundamentos & $51.13 \%$ & $58.55 \%$ & $79.23 \%$ \\
Unidad 2: Expresiones algebraicas & $36.59 \%$ & $44.99 \%$ & $66.42 \%$ \\
Unidad 3: Ecuaciones lineales y desigualdades & $30.01 \%$ & $38.58 \%$ & $55.70 \%$ \\
Unidad 4: Gráfíca de línea y pendiente & $15.87 \%$ & $29.15 \%$ & $41.08 \%$ \\
Unidad 5: Sistemas de ecuaciones & $17.43 \%$ & $27.75 \%$ & $40.03 \%$ \\
Unidad 6: Expresiones con exponente & $18.48 \%$ & $29.19 \%$ & $46.34 \%$ \\
Unidad 7: Cuadrática y polinomios & $21.60 \%$ & $31.46 \%$ & $48.09 \%$ \\
Unidad 8: Geometría y ecuaciones & $19.80 \%$ & $31.75 \%$ & $47.35 \%$ \\
\hline
\end{tabular}


La Tabla 2 expone el comparativo entre los índices de aprobación, reprobación, abandono y desempeño académico en función de que los estudiantes hayan o no realizado el curso PREIN. Para el desempeño academico se utilizó la escala de medición colombiana de 0 a 5 puntos, con una nota mínita de aprobación de 3. Los datos indican mejores tasas de aprobación para los estudiantes que realizaron el curso durante las tres implementaciones. Además, se observa que en las tres implementaciones abandonaron menos estudiantes que realizaron el curso y que el desempeño academico promedio de los estudiantes que realizaron el curso fue superior a los que no lo realizaron con una diferencia de 4 décimas en la primera implementación, 5 décimas en la segunda implementación y 9 décimas en la tercera implementación.

Tabla 2 Tasas de aprobación, reprobación, deserción y desempeño academico

\begin{tabular}{|c|c|c|c|c|c|c|}
\hline & \multicolumn{2}{|c|}{$\begin{array}{c}\text { Primera } \\
\text { Implementación } \\
\text { 2018-II }\end{array}$} & \multicolumn{2}{|c|}{$\begin{array}{c}\text { Segunda } \\
\text { Implementación } \\
2019-\mathrm{I}\end{array}$} & \multicolumn{2}{|c|}{$\begin{array}{c}\text { Tercera } \\
\text { Implementación } \\
\text { 2019-II }\end{array}$} \\
\hline & PREIN & NO PREIN & PREIN & NO PREIN & PREIN & NO PREIN \\
\hline & $\mathrm{N}=522$ & $\mathrm{~N}=1781$ & $\mathrm{~N}=1947$ & $\mathrm{~N}=2688$ & $\mathrm{~N}=1572$ & $\mathrm{~N}=2396$ \\
\hline Aprobaron & $76 \%$ & $62 \%$ & $75 \%$ & $57 \%$ & $76 \%$ & $52 \%$ \\
\hline Reprobaron & $17 \%$ & $13 \%$ & $23 \%$ & $39 \%$ & $14 \%$ & $31 \%$ \\
\hline Abandonaron & $7 \%$ & $25 \%$ & $2 \%$ & $4 \%$ & $10 \%$ & $17 \%$ \\
\hline Desempeño académico promedio & 3.3 & 2.9 & 3.3 & 2.8 & 3.6 & 2.7 \\
\hline
\end{tabular}

El p-valor en la prueba Chi-Cuadrado para los tratamientos PREIN y No PREIN durante los semestres cadademicos 2018-II, 2019-I y 2019-II fue, respectivamente, 3.9E-18, 1.5E-35 y 9.6E-52, es decir, prácticamente cero, por lo tanto se rechaza la hipótesis nula de independencia entre las variables de estudio, es decir, las tasas de aprobación, perdida y cancelación dependen de que el estudiante de nuevo ingreso realice o no el curso PREIN.

También se analizaron los resultados considerando la variable docente. Para ello, se midió por cada docente el porcentaje de matriculados al curso PREIN, el porcentaje de avance total en PREIN y el porcentaje de estudiantes que aprobaron la asignatura. Se estableció una escala de medición para los porcentajes que permite distinguir cuatro niveles: Bajo (de $0 \%$ a $30 \%$ ), Básico (de $31 \%$ a $59 \%$ ), Sobresaliente (de $60 \%$ a $79 \%$ ) y Excelente (de $80 \%$ a $100 \%$ ). Respecto al porcentaje de estudiantes matriculados en el PREIN por docente, la Tabla 3 muestra que los resultados mejoraron considerablemente de la primera a la segunda implementación, así por ejemplo, en la primera implentación el porcentaje de docentes en el nivel bajo fue $81 \%$ mientras que en la segunda implentación para ese mismo nivel el porcentaje fue del $5 \%$. Paralelamente, aumentan los docentes con porcentajes de matriculados en PREIN en los tramos sobresaliente y excelente. Anque en la tercera implementación los resultados del número de matriculados no fue igual de favorable que en la segunda, se evidencia que comparados con los resultados de la primera implentación si reflejan mejoras. Lo anterior, debido a que semestre a semestre existe variación en la carga docente por diferentes factores. 


\begin{tabular}{cccc}
\hline & \multicolumn{3}{c}{ Docentes } \\
\cline { 2 - 4 } $\begin{array}{c}\text { Porcentaje de estudiantes } \\
\text { matriculados en PREIN }\end{array}$ & $\begin{array}{c}\text { Primera } \\
\text { implementación } \\
\mathrm{N}=37\end{array}$ & $\begin{array}{c}\text { Segunda Implementación } \\
\mathrm{N}=59\end{array}$ & $\begin{array}{c}\text { Tercera Implementación } \\
\mathrm{N}=56\end{array}$ \\
\hline Bajo [0 \% a 30 \%] & $81 \%$ & $5 \%$ & $46 \%$ \\
Básico [31 \% a 59\%] & $14 \%$ & $41 \%$ & $27 \%$ \\
Sobresaliente [60 \% a 79 \%] & $5 \%$ & $37 \%$ & $21 \%$ \\
Excelente [80 \% a 100 \%] & $0 \%$ & $17 \%$ & $5 \%$ \\
\hline
\end{tabular}

En relación al porcentaje de avance total en el curso PREIN obtenido por los estudiantes de cada docente, durante la primera y segunda implementación se obtuvieron los resultados que recoge la Tabla 4. Estos datos evidencian una disminución del $39 \%$ en el nivel bajo de la segunda implementación comparado con el obtenido en la primera implementación. También se constata que, tras la segunda implementación, aparecen por primera vez docentes cuyo alumnado avanza en niveles sobresalientes e incluso excelentes.

Tabla 4. Porcentaje de profesorado en cada tramo de la escala, teniendo en cuenta el porcentaje de avance en PREIN por docente.

\begin{tabular}{cccc}
\hline & \multicolumn{3}{c}{ Docentes } \\
\cline { 2 - 4 } $\begin{array}{c}\text { Porcentaje de avance de los } \\
\text { estudiantes en PREIN }\end{array}$ & $\begin{array}{c}\text { Primera } \\
\text { implementación } \\
\mathrm{N}=37\end{array}$ & $\begin{array}{c}\text { Segunda } \\
\text { Implementación } \\
\mathrm{N}=59\end{array}$ & $\begin{array}{c}\text { Tercera } \\
\text { Implementación } \\
\mathrm{N}=56\end{array}$ \\
\hline Bajo [0\% a 30\%] & $81 \%$ & $42 \%$ & $14 \%$ \\
Básico [31\% a 59\%] & $19 \%$ & $42 \%$ & $46 \%$ \\
Sobresaliente [60\% a 79\%] & $0 \%$ & $14 \%$ & $34 \%$ \\
Excelente [80 \% a 100\%] & $0 \%$ & $2 \%$ & $5 \%$ \\
\hline
\end{tabular}

Respecto al porcentaje de estudiantes que aprobaron la asignatura por docente la Tabla 5 registra que de la primera a la tercera implementación hubo un aumento en los niveles sobresaliente y excelente, así como una disminución en los niveles bajo y básico.

Tabla 5. Porcentaje de profesorado en cada tramo de la escala, teniendo en cuenta el porcentaje de aprobación por docente

\begin{tabular}{|c|c|c|c|}
\hline \multirow{2}{*}{$\begin{array}{l}\text { Porcentaje de estudiantes que } \\
\text { aprueban la asignatura }\end{array}$} & \multicolumn{3}{|c|}{ Docentes } \\
\hline & $\begin{array}{c}\text { Primera } \\
\text { implementación } \\
\mathrm{N}=37\end{array}$ & $\begin{array}{c}\text { Segunda } \\
\text { Implementación } \\
\mathrm{N}=59\end{array}$ & $\begin{array}{c}\text { Tercera } \\
\text { Implementación } \\
\mathrm{N}=56 \\
\end{array}$ \\
\hline Bajo $[0 \%$ a $30 \%]$ & $11 \%$ & $5 \%$ & $5 \%$ \\
\hline Básico [31 \% a $59 \%$ ] & $51 \%$ & $41 \%$ & $38 \%$ \\
\hline Sobresaliente $[60 \%$ a $79 \%$ ] & $24 \%$ & $37 \%$ & $41 \%$ \\
\hline Excelente $[80 \%$ a $100 \%$ ] & $14 \%$ & $17 \%$ & $16 \%$ \\
\hline
\end{tabular}

(c)) EY-NC-ND 2020, Universitat Politècnica de València

Congreso In-Red (2020) 
Además, con los resultados obtenidos por cada docente se midio el nivel de asociación entre las variables: porcentaje de matriculados en PREIN, porcentaje de avance total en PREIN y porcentaje de aprobación, encontrándose (Tabla 6) correlación positiva y fuerte entre el porcentaje de matriculados en PREIN y el porcentaje de avance en PREIN, es decir, los docentes que logran que la mayoría de sus estudiantes se matriculen en el curso también logran que ellos obtengan un mayor porcentaje de progreso en el curso. Sin embargo, no se aprecia apenas correlación entre las otras dos combinaciones de porcentajes, es decir, no parece haber una asociación clara entre el porcentaje de matriculados en PREIN y el porcentaje de aprobación para cada docente, ni entre este porcentaje y el de avance en PREIN.

Tabla 6. Coeficiente de correlación

\begin{tabular}{lccc}
\hline & \% matriculados PREIN & $\%$ avance PREIN & $\%$ aprobación \\
\hline$\%$ matriculados PREIN & 1 & & \\
$\%$ avance PREIN & 0.78 & -0.06 & 1 \\
$\%$ aprobación & 0.04 & & \\
\hline
\end{tabular}

\section{Conclusiones}

El uso de la plataforma KHAN ACADEMY posibilita que las IES brinden un entrenamiento virtual a los estudiantes de nuevo ingreso con el fin de fortalecer los presaberes matemáticos de forma online y sin generar altos costos. El análisis conjunto de las Tablas 2-5 evidencia una mejora de todos los indicadores de rendimiento.

Como mecanismo de fortalecimiento de la estrategia PREIN se hace indispensable el rol del docente para llevar a cabo el cumplimiento de los objetivos del curso. Mediante la capacitación docente y propiciando una motivación activa en el aula de clase los docentes logran incrementar el número de matriculados y el porcentaje de progreso en el curso PREIN.

Es cierto que los índices globales de los docentes mejoraron de la primera a la segunda implementación, sin embargo, hasta el momento no podemos asegurar que exista una relación entre el porcentaje de aprobación y el porcentaje de avance en el curso PREIN, cuando se realiza el análisis docente a docente. Lo anterior puede ser debido a la forma en que el docente promueve el curso en el aula mediante los diferentes incentivos que otorga o por los diferentes mecanismos de evaluación que emplea sustentado en el derecho a la libertad de cátedra.

En futuras investigaciones se medirá el impacto que ha generado el curso en el desempeño academico de los estudiantes en las asignaturas del área de matemática, mediante análisis comparativo de las tasas de retención, aprobación y desempeño académico obtenidas tras la implementación del curso, con las de semestres anteriores.

\section{Referencias}

AGUILAR, G. (2014). "Influencia de los dominios conceptuales en las competencias académicas: área de matemátic s para ingenierías en Revista Educación en Ingeniería, 9(18), p. 74-88.

DÍAZ, P. y RODRÍGUEZ-MUÑIZ, L.J. (2013). "Experiences in Spanish Universities to reduce failure in Mathematics of incoming students" en Procedia-Social and Behavioral Sciences, 93, p. 2006-2009. 
DICHEVA, D. DICHEV, C. AGRE, G. y ANGELOVA, G. (2015). "Gamification in education: a systematic mapping study" en Journal of Educational Technology \& Society, 18(3), p. 75-94.

MARTÍNEZ, P. PÉREZ, J. y MARTÍNEZ, M. (2016). "Las TICS y el entorno virtual para la tutori universitaria” en Educación XXI, 19(1), p. 287-310.

Ministerio de Educación Nacional de Colombia MEN. Estadísticas del Sistema para la Prevención de la Deserción de la Educación Superior. $\quad<$ https://www.mineducacion.gov.co/sistemasinfo/spadies/InformacionInstitucional/357549:Estadisticas-de-Desercion> [Consulta: 14 de febrero de 2020]

MURCIA, M. y HENAO, J. (2015). "Educación matemátic en Colombia, una perspectiva evolucionaria" en Entre Ciencia e Ingeniería, 9(18), p. 23-30.

OCDE. Resultados PISA 2018 Los jóvenes están luchando en el mundo digital. $<$ http:/www.oecd.org/centrodemexico/los-jovenes-estan-luchando-en-el-mundo-digital-dice-la-mas-recienteedicion-de-pisa-de-la-ocde.htm> [Consulta: 1 de febrero de 2020]

RAMÍREZ, M. y VIZCARRA, J. (2016). "Desarrollo de habilidades matemáti as en estudiantes normalistas mediante Khan Academy” en Ra Ximhai, 12(6), p. 285-293.

RODRÍGUEZ, G. GÓMEZ, V. y ARIZA, M. (2014). "Calidad de la educación superior a distancia y virtual: un análisi de desempeño académico en Colombia” en Investigación \& Desarrollo, 22(1), p. 79-120.

RODRÍGUEZ-MUÑIZ, L.J. y DÍAZ, P. (2015). "Estrategias de las universidades españolas para mejorar el rendimiento en matemá icas del alumnado de nuevo ingreso" en Aula abierta, 43(2), p. 69-76.

UTOMO, H. BON, A. y HENDAYUN, M. (2018, July). "The integrated academic information system support for education 3.0 in higher education institution: Lecturer perspective" en Journal of Physics: Conference Series 1049(1), p. 012102. IOP Publishing.

VALDÉS, A. ANGULO, J. URÍAS, M. GARCÍA, R. y MORTIS, S. (2011). "Necesidades de capacitación de docentes de educación básica en el uso de las TIC" e Pixel-Bit Revista de Medios y Educación, 39(1), p. 211-223. 